\title{
Isquemia aguda por trombosis de arteria poplítea tras luxación anterior de rodilla
}

\author{
Martínez-Izquierdo A. ${ }^{1}$, Sáinz-González F. ${ }^{2}$, Abu-Sniemeh A.A. ${ }^{3}$ \\ Sanid. mil. 2021; 77 (2): 90-93, ISSN: 1887-8571
}

\begin{abstract}
RESUMEN
Las luxaciones de rodilla son poco frecuentes pero en un elevado porcentaje pueden estar asociadas a graves complicaciones neurovasculares. La afectación del nervio peroneo común es la lesión más frecuente. La arteria poplítea está afectada principalmente en los casos de luxación posterior y en traumatismos con afectación multiligamentosa. Presentamos un caso de luxación anterior tibio-femoral acompañada de isquemia aguda por oclusión de la arteria poplítea, que precisó cirugía de revascularización mediante by pass. La evolución postoperatoria fue satisfactoria, consiguiendo la recuperación de pulsos pedio y tibial posterior. Se precisa un alto grado de sospecha clínica para la detección de las complicaciones vasculares. La demora en el tratamiento quirúrgico arterial aumenta gravemente el riesgo de amputación.
\end{abstract}

PALABRAS CLAVE: luxación, rodilla, arteria poplítea, isquemia, by pass femoropopliteo

Acute ischemia due to popliteal artery thrombosis after anterior knee dislocation

SUMMARY

Knee dislocations are rare but in a high percentage may cause severe and devastating neurovascular complications. Damage of the common peroneal nerve is the most frequent injury. The popliteal artery is more prone to be affected in posterior knee dislocation and whithin multiligament traumatic injury. We present a case of anterior tibio-femoral dislocation associated with acute ischemia due to popliteal artery occlusion that needed emergent surgical intervention for revascularisation of the left lower limb through bypass. Postoperative outcome was satisfactory with pedal and posterior tibial pulses recovery. A high grade of clinical suspicion is needed for early detection of vascular complications. Delay in vascular intervention increases dramatically the risk of irreversible injury and the need of amputation.

KEY WORDS: Dislocation, knee, popliteal artery, ischemia, femoro-popliteal by pass

\section{INTRODUCCIÓN}

La luxación de rodilla es una lesión muy poco frecuente, por debajo del $0.5 \%$ de todas las luxaciones y con una incidencia menor del $0.2 \%$ de las urgencias traumatológicas ${ }^{1}$.

Se clasifica de acuerdo a criterios anatómicos en anterior, posterior, interna o externa según el desplazamiento de la tibia respecto al fémur. De todas éstas la más frecuente es la anterior hasta en un $40 \%$ de los $\operatorname{casos}^{2}$.

Habitualmente las luxaciones de rodilla se acompañan de lesiones múltiples ligamentosas, meniscales, fracturas y lesiones neurovasculares ${ }^{3}$.

El nervio peroneo común o ciático poplíteo externo (CPE) presenta alteraciones en una cuarta parte de los casos.

La arteria poplítea es el vaso más frecuentemente afectado por su disposición anatómica: fijación vascular anatómica pro-

\footnotetext{
1. Teniente coronel médico. Servicio de Angiología y Cirugía Vascular. Hospital Central de la Defensa "Gómez Ulla". Madrid.

2. Coronel médico. Servicio de Angiología y Cirugía Vascular. Hospital Central de la Defensa "Gómez Ulla". Madrid. 3. Médico especialista. Servicio de Angiología y Cirugía Vascular. Hospital Central de la Defensa "Gómez Ulla". Madrid
}

Dirección para correspondencia: Antonio Martínez Izquierdo. Hospital Central de la Defensa "Gómez Ulla". Glorieta del Ejercito s/n 28047 Madrid. amarizq@oc.mde.es

Recibido: 18 de noviembre de 2020

Aceptado: 20 de febrero de 2021

doi: $10.4321 /$ S1887-85712021000200004 ximal en hiato de los abductores y distal en el arco tendinoso del Soleo. Esto ocasiona una reducción de su movilidad y en las luxaciones de tibia se produce la tracción o rotura arterial en esos puntos de fijación ${ }^{4}$.

Las lesiones intravasculares por compresión son la disección intimal con la formación de flap oclusivo, la trombosis in situ o la rotura arterial ${ }^{5}$.

Dada la importancia pronóstica de la lesión arterial se debe realizar una valoración vascular en el momento del diagnóstico de luxación y tras su reducción.

La cirugía vascular en estos casos tiene carácter de urgencia dado que la demora en el tratamiento de la isquemia arterial conduce a lesiones irreversibles, que conllevan a la amputación por encima de la rodilla en muchos de los casos.

A propósito de un caso tratado en nuestra institución se realiza una descripción del procedimiento llevado a cabo y una breve revisión de esta patología.

\section{CASO CLÍNICO}

Paciente varón de 62 años que es trasladado al Servicio de Urgencias por el SAMUR tras caída accidental por un tramo de 3 escaleras hace 5 horas. Presenta dolor de reposo, impotencia funcional y marcada deformidad de rodilla izquierda.

No refiere antecedentes medicoquirúgicos de interés ni alergias medicamentosas conocidas. 


\section{Isquemia aguda por trombosis de arteria poplítea tras luxación anterior de rodilla}

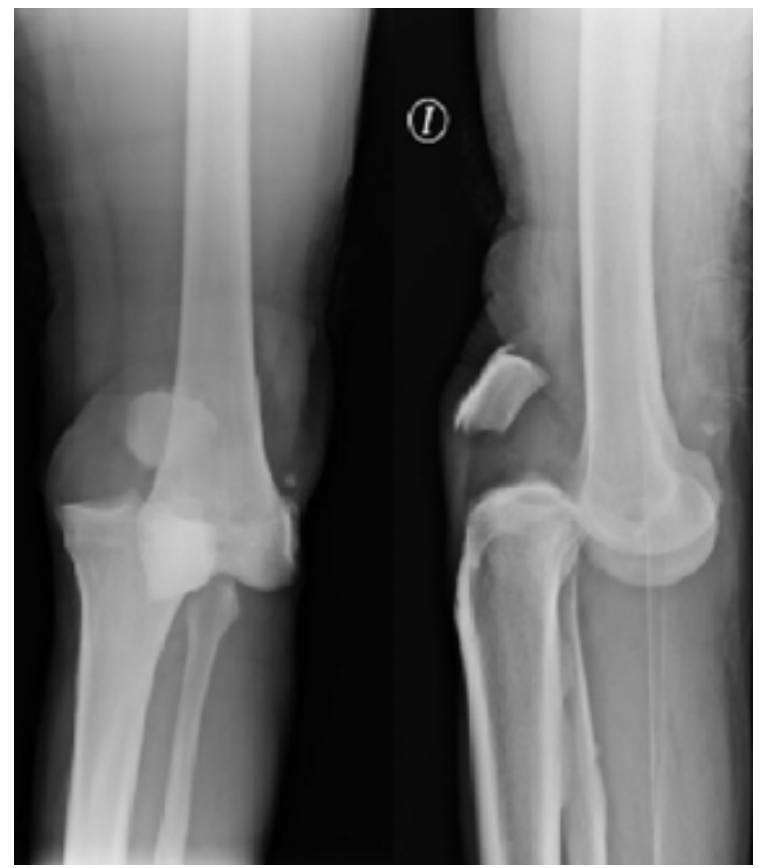

Fig 1. Radiografía AP y lateral

Se realiza radiografía anterior y lateral de $\mathrm{Rx}$ rodilla izquierda (Fig 1) confirmando la sospecha clínica: Luxación Femorotibial anterior e interna.

Con carácter urgente y bajo sedo-analgesia se procede a la reducción cerrada de la luxación de rodilla.

Tras reducción se aprecia frialdad distal en antepie y dedos por lo que se sospecha afectación vascular..

A la exploración presenta ausencia de pulso poplíteo y de vasos distales con frialdad desde tercio medio de pierna y edema aunque conserva sensibilidad. La movilidad no es valorable por la férula de fijación. Refiere dolor a nivel gemelar en reposo y a la presión.

En miembro inferior contralateral (MID) presenta una exploración neurovascular dentro de la normalidad.

Se realiza estudio ecodoppler arterial y venoso de MII apreciando ausencia de flujo desde la arteria poplítea en $1^{a}$ porción. La oclusión se sitúa distal a una arteria genicular.

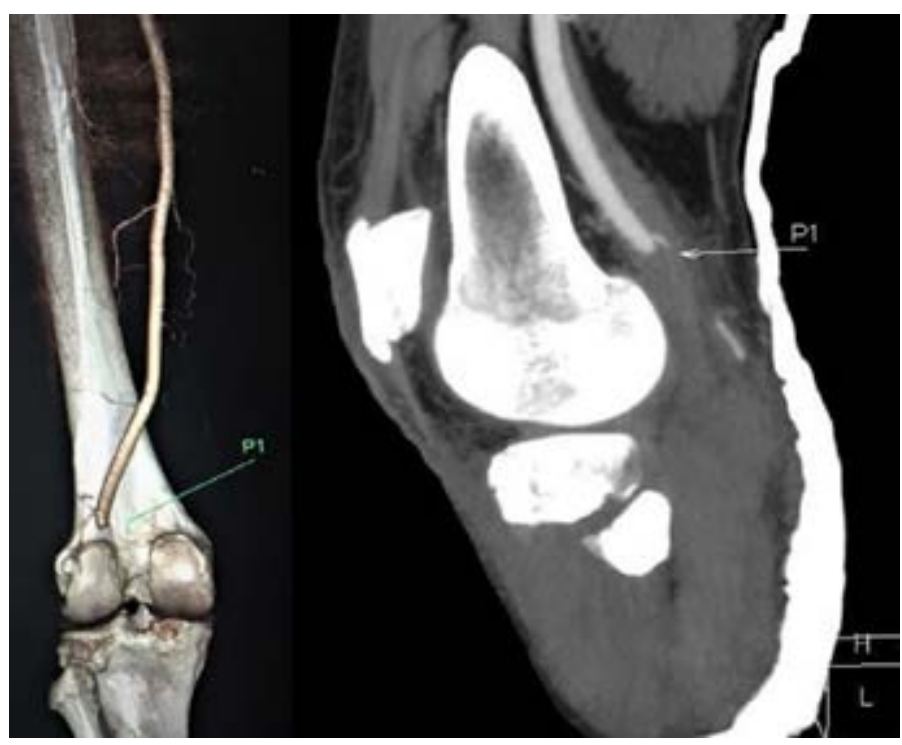

Fig 2. AngioTC: Reconstrucción - Imagen sagital
No presenta signos ecográficos de TVP y la vena safena interna está dilatada y varicosa.

Ante la situación de isquemia aguda por trombosis traumática de arteria poplítea y con el fin de planificar la cirugía se realiza AngioTAC (Fig 2) de MII, confirmando oclusión poplítea desde $1^{\mathrm{a}}$ porción (flecha P1) y unicamente se identifica distalmente flujo en tibial anterior (Ta).

Ante los hallazgos clínicos y el tiempo evolutivo desde el traumatismo se decide la intervención quirúrgica urgente. Se realiza trombectomía proximal y distal mediante abordaje poplíteo infragenicular obteniendo abundante material trombótico en la arteria poplítea, en Ta y tronco tibioperoneo (TTP).

Proximalmente presenta flujo no pulsátil, por lo que ante sospecha de disección arterial, y tras abordaje femoral distal y control arterial con Vessel-loops, se realiza by pass desde primera hasta tercera porción de arteria poplítea con injerto tubular de 6 mm de PTFE anillado, en disposición anatómica, consiguiendo la revascularización de MII. (Fig 3)

Posteriormente es intervenido por el servicio de Traumatología para la estabilización de la rodilla, colocando fijador externo con dos pines lateromediales de $6 \times 200 \mathrm{~mm}$ en fémur distal y 2 pines de $6 \times 160 \mathrm{~mm}$ en cara anteromedial de tercio medio de tibia y montaje con tres barra de titanio.

Ante la inestabilidad de la rodilla y con vistas a la estabilización quirúrgica en una segunda intervención, se realiza RMN apreciando rotura completa del ligamento cruzado anterior (LCA), rotura completa-rotura de alto grado del ligamento cruzado posterior (LCP) y esguince grado II en la región proximal del ligamento colateral externo. El ligamento colateral interno y meniscos no presentan imágenes de rotura. No hay imágenes de fractura ni edema óseo. Presenta derrame articular severo con pequeño quiste de Baker. La rótula y cartílago femoropatelar no se detectan lesiones.

Es dado de alta con presencia de pulsos pedio y tibial posterior con buena temperatura distal, pendiente de reconstrucción multiligamentaria.

Se aprecia limitación para la flexión dorsal del tobillo y disminución de la fuerza $3+/ 5$; todo ello compatible con lesión del nervio peroneo común.

\section{DISCUSIÓN}

A pesar de que la luxación de rodilla es muy infrecuente, las posibles lesiones vasculares y neurológicas asociadas tienen una repercusión muy importante para la funcionalidad y viabilidad de la extremidad.

Se pueden presentar complicaciones debido a la oclusión de la arteria poplítea, por afectación del nervio peroneo común y menos frecuentemente por trombosis venosa profunda (TVP) o generar un síndrome compartimental ${ }^{3}$.

Se ha estimado que se presentan lesiones vasculares entre el $28 \%$ to $46 \%$ de los casos de luxación ${ }^{6}$.

La clínica de isquemia aguda se produce por disección intimal y trombosis o incluso en forma de hemorragia aguda o masa pulsátil por formación de pseudoaneurisma tras rotura arterial ${ }^{7}$.

El nervio peroneo común puede presentar lesiones en un $25 \%$ de los casos que van desde neuroapraxia hasta neurotmesis. La 
Fig 3. Imagen quirúrgica del by pass

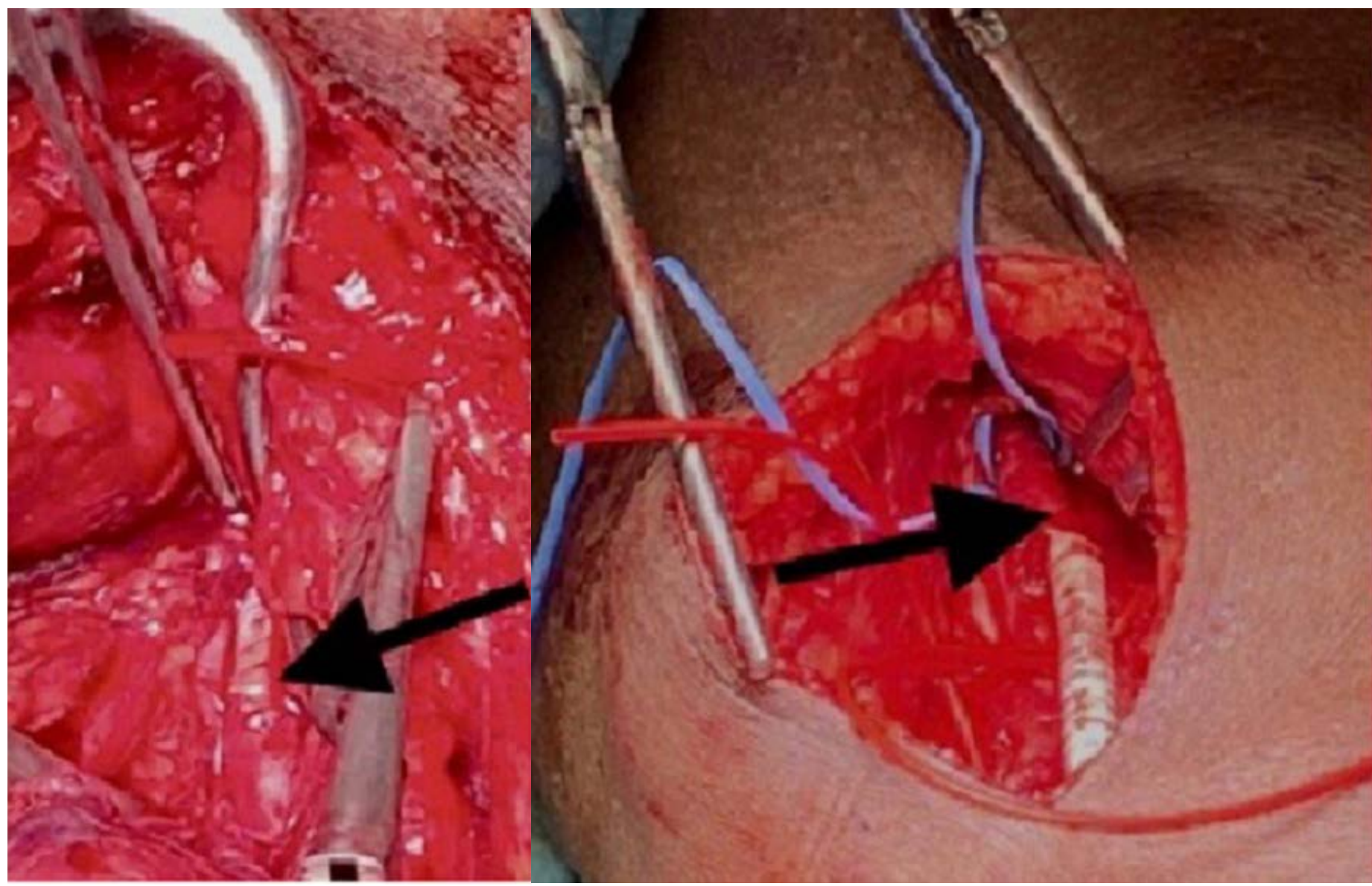

Anastomosis distal

Anastomosis proximal

evolución clínica de las lesiones en la mayoría de los pacientes es la recuperación completa, pero los casos que presentan una parálisis completa menos del $40 \%$ recuperan la dorxiflexión del tobillo ${ }^{8}$.

Las luxaciones con afectación multiligamentosa presentan una mayor incidencia de complicaciones asociadas. Las lesiones que afectan a ambos ligamentos cruzados y ligamento colateral, tanto en luxaciones anteriores como posteriores, presentan una elevada incidencia de lesión vascular debido a la inestabilidad de la articulación? .

El diagnóstico de la complicación vascular no siempre es sencillo. Muchas veces debido a la deformidad de la extremidad y al edema asociado, no es posible la palpación de pulsos y la clínica no es muy florida. La analgesia recibida para la reducción de la luxación y la presencia de ramas arteriales geniculares procedentes de la arteria descendente de la rodilla y de la tercera perforante de la arteria femoral profunda, mantienen un mínimo flujo distal a la oclusión de la arteria poplítea que ocultan los clásicos síntomas de isquemia aguda; dolor, palidez, parestesia, parálisis y ausencia de pulsos.

Ante luxaciones de rodilla que presentan clínica de isquemia o ausencia de pulsos pedio o tibial posterior previamente al traumatismo o tras reducción de la misma, se debe sospechar lesión vascular asociada.
El diagnóstico de confirmación se basa en estudios de imagen. Algunos autores preconizan el uso sistemático de arteriografía o angioTC en todos los casos de luxación de rodilla ${ }^{10}$.

En nuestro servicio de Angiología y Cirugía Vascular ante casos que presenten clínica de isquemia o exploración vascular anómala utilizamos de rutina la exploración ecográfica duplex para realizar un screening vascular. Con ello logramos el diagnóstico y la valoración venosa para una posible intervención quirúrgica de revascularización.

La necesidad de restaurar el flujo arterial se ha estimado en el $80 \%$ de los casos de luxación con afectación vascular. La demora en la intervención mayor de las 8 horas conduce a la amputación en más del $85 \%$ de los casos ${ }^{11}$.

La cirugía de revascularización requiere en la mayoría de los casos la realización de by pass, puesto que la trombectomía simple resulta insuficiente dado el mecanismo de lesión ${ }^{12}$.

La utilización de vena safena interna (VSI) ipsilateral es lo recomendado en estos procedimientos dada la resistencia a la infección, su flexibilidad permitiendo la elongación con la flexión y la vasodilatación comparado con los materiales sintéticos de las prótesis vasculares; Politetrafluoruroetileno expandido (PTFEe) o dacron.

No se debe utilizar la VSI si presenta signos ecográficos de TVP o si presenta alteraciones varicosas. 


\section{Isquemia aguda por trombosis de arteria poplítea tras luxación anterior de rodilla}

En nuestro caso la presencia de severa dilatación venosa de la VSI nos condujo a optar por material protésico PTFEe reforzado.

La realización de fasciotomías en los cuatro compartimentos dependerá de la clínica y del tiempo de instauración de la isquemia. En nuestro caso dado que se pudo realizar la intervención con una demora menor a 2 horas no precisó la fasciotomía terapeútica ni profiláctica.

El diagnóstico precoz y el tratamiento quirúrgico de las lesiones de la arteria poplítea en el contexto de una luxación de rodilla son primordiales para lograr la funcionalidad de la extremidad y la viabilidad de la misma.

No existe un consenso de actuación en los casos de luxación de rodilla pero consideramos que el seguimiento clínico en las primeras horas tras el traumatismo y tras la reducción de la luxación son primordiales y debe realizarse una valoración vascular continuada en el tiempo para poder detectar lesiones ocultas ante la sintomatología florida de la propia luxación.

\section{BIBLIOGRAFÍA}

1. Robertson A, Nutton RW, Keating JF. Dislocation of the knee. J Bone Joint Surg [Br] 2006;88-B:706-11.

2. Henrichs A. A review of knee dislocations. J Athl Train. 2004;39:365-369.
3. Peskun CJ, Levy BA, Fanelli GC, Stannard JP, Stuart MJ, MacDonald PB, et al. Diagnosis and management of knee dislocations. Phys Sportsmed. 2010;38(4):101-11. doi: 10.3810/psm.2010.

4. Dwyer T, Whelan D. Anatomical considerations in multiligament knee injury and surgery. J Knee Surg. 2012;25:263-274

5. A.Chapman. Popliteal artery damage in closed injuries of the knee.The Journal of Bone \& Joint Surgery - BritishVolume,vol. 67,no.3,pp.420-423,1985,6

6. H. L. Steele and A. Singh, Vascular injury after occult knee dislocation presenting as compartment syndrome, The Journal of Emergency Medicine,vol .42, no.3,pp. $271-274,2012$

7. Rodríguez Moro C, Sáinz González F, Pérez-Piqueras Gómez A. Rotura completa de arteria poplítea de un mes de evolución, tras meniscectomía parcial artroscópica. Revista española de cirugía ortopédica y traumatología, ISSN 1888-4415, Vol. 56, Nº. 2, 2012, págs. 153-155

8. Jarret M. Woodmass · Nicholas P. J. Romatowski · John G. Esposito · Nicholas G. H. Mohtadi· Peter D. Longino. A systematic review of peroneal nerve palsy and recovery following traumatic knee dislocation. Knee Surg Sports Traumatol Arthrosc (2015) 23:2992-3002 DOI 10.1007/s00167-015-3676-7

9. Treiman GS, Yellin AE, Weaver FA, Wang S, Ghalambor N, Barlow W, Snyder B, Pentecost MJ. Examination of the patient with a knee dislocation. The case for selective arteriography. Arch Surg. 1992;127:1056-1062; discussion 1062-1063

10. Klineberg EO, Crites BM, Flinn WR, Archibald JD, Moorman CT. The role of arteriography in assessing popliteal artery injury in knee dislocations. J Trauma. 2004;56(4):786-90. doi: 10.1097/01.ta.0000075346.05460.d6

11. Rihn JA, Groff YJ, Harner CD, Cha PS. The acutely dislocated knee: evaluation and management. J Am Acad Orthop Surg. 2004;12(5):334-46. 5

12. J. S. Wilson, A. Miranda, B. L. Johnson, M. L. Shames, M. R. Back, and D. F. Bandyk, Vascular injuries associated with elective orthopedic procedures. Annals of Vascular Surgery,vol. 17,no.6,pp.641-644,2003 